Supplement of Atmos. Chem. Phys., 21, 10825-10849, 2021

https://doi.org/10.5194/acp-21-10825-2021-supplement

(c) Author(s) 2021. CC BY 4.0 License.

(c) (1)
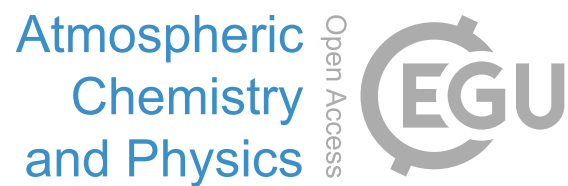

Supplement of

\title{
Measured and modelled air quality trends in Italy over the period 2003-2010
}

Ilaria D'Elia et al.

Correspondence to: Ilaria D’Elia (ilaria.delia@enea.it)

The copyright of individual parts of the supplement might differ from the article licence. 


\section{SUPPLEMENTARY MATERIAL}

\section{Supplementary S1}

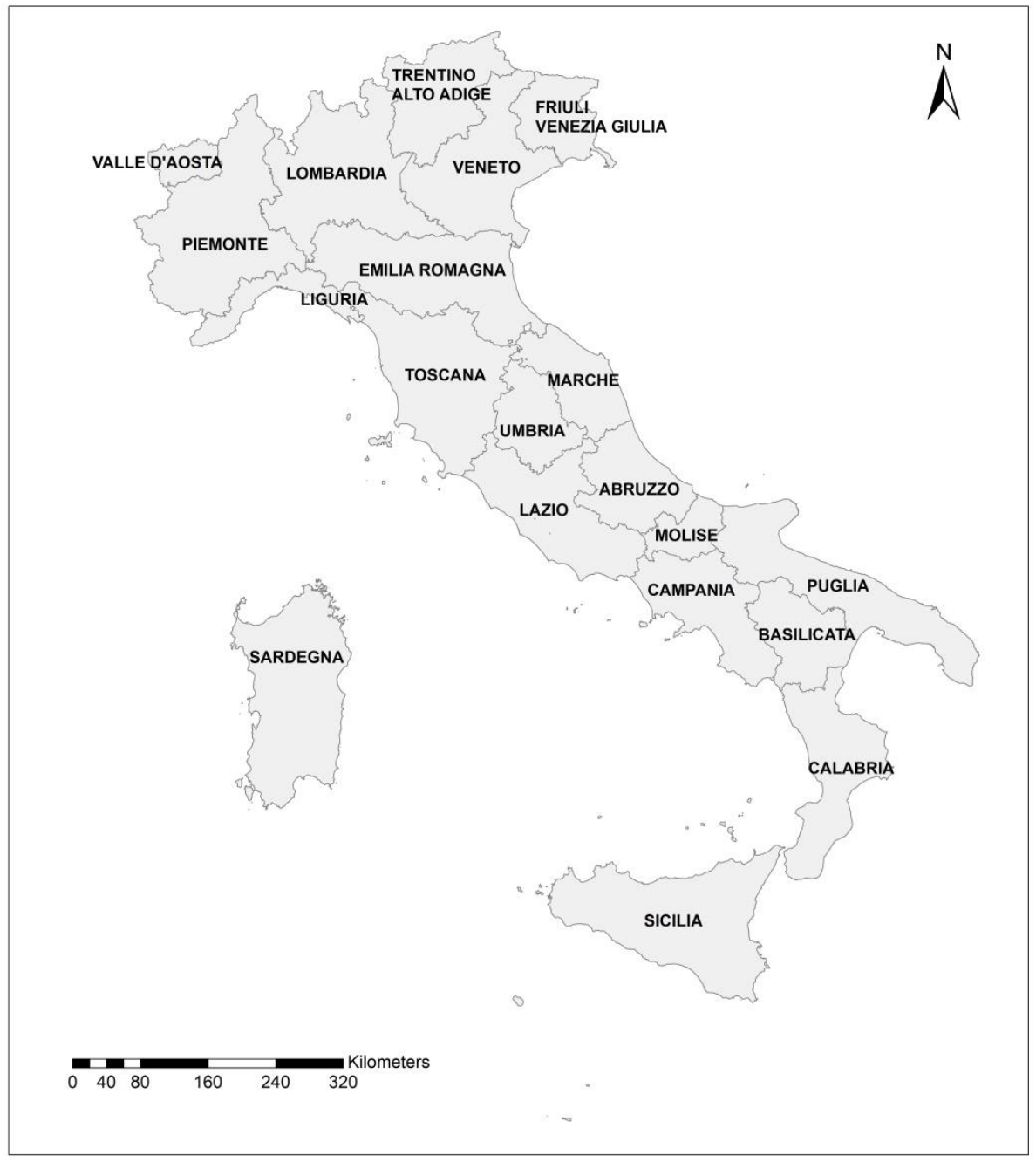

Figure S1 - Map of the Italian administrative Regions. 

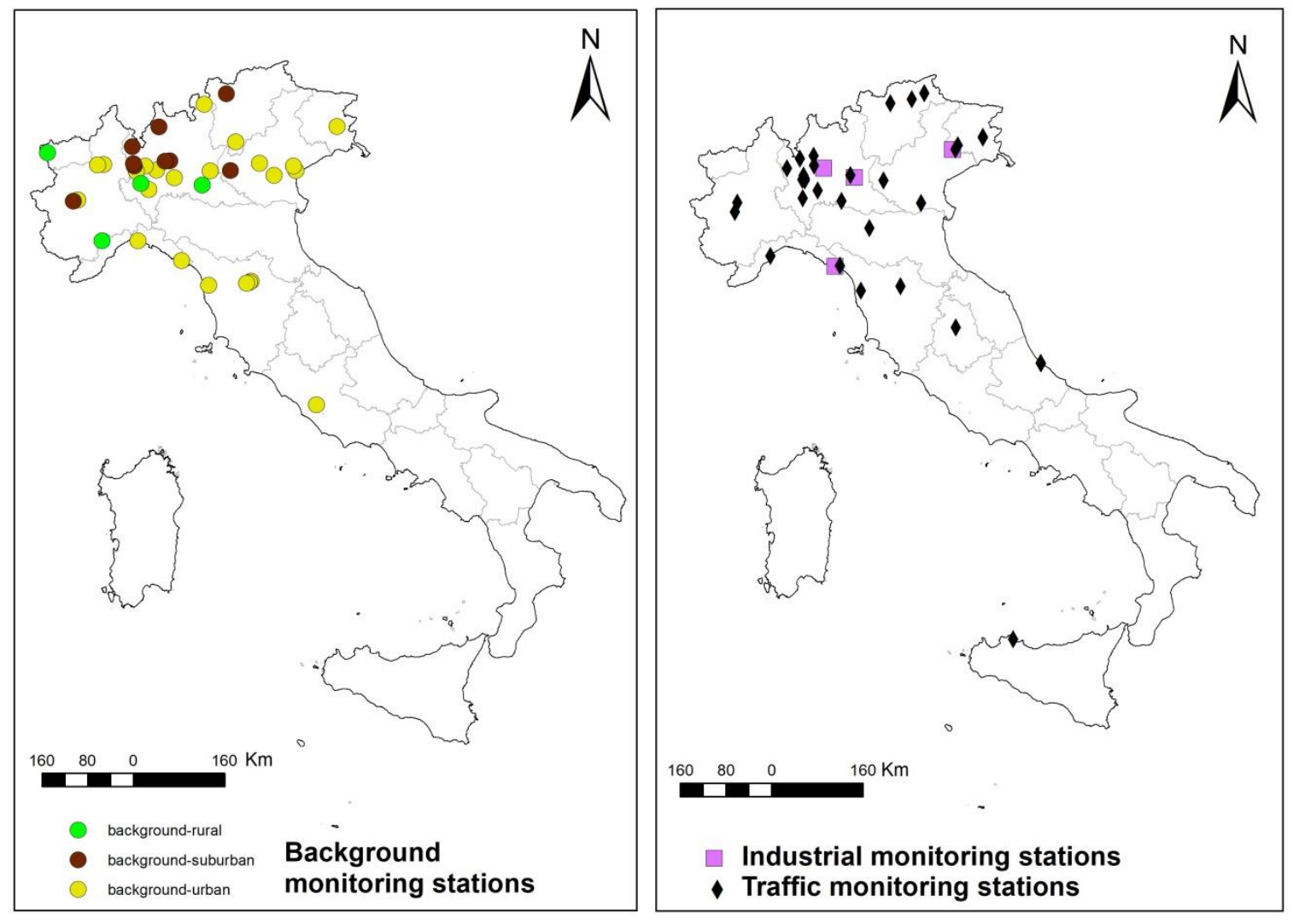

Figure S2 - Map of the Italian sites monitoring $\mathrm{NO}_{2}$ concentrations considered for the study.
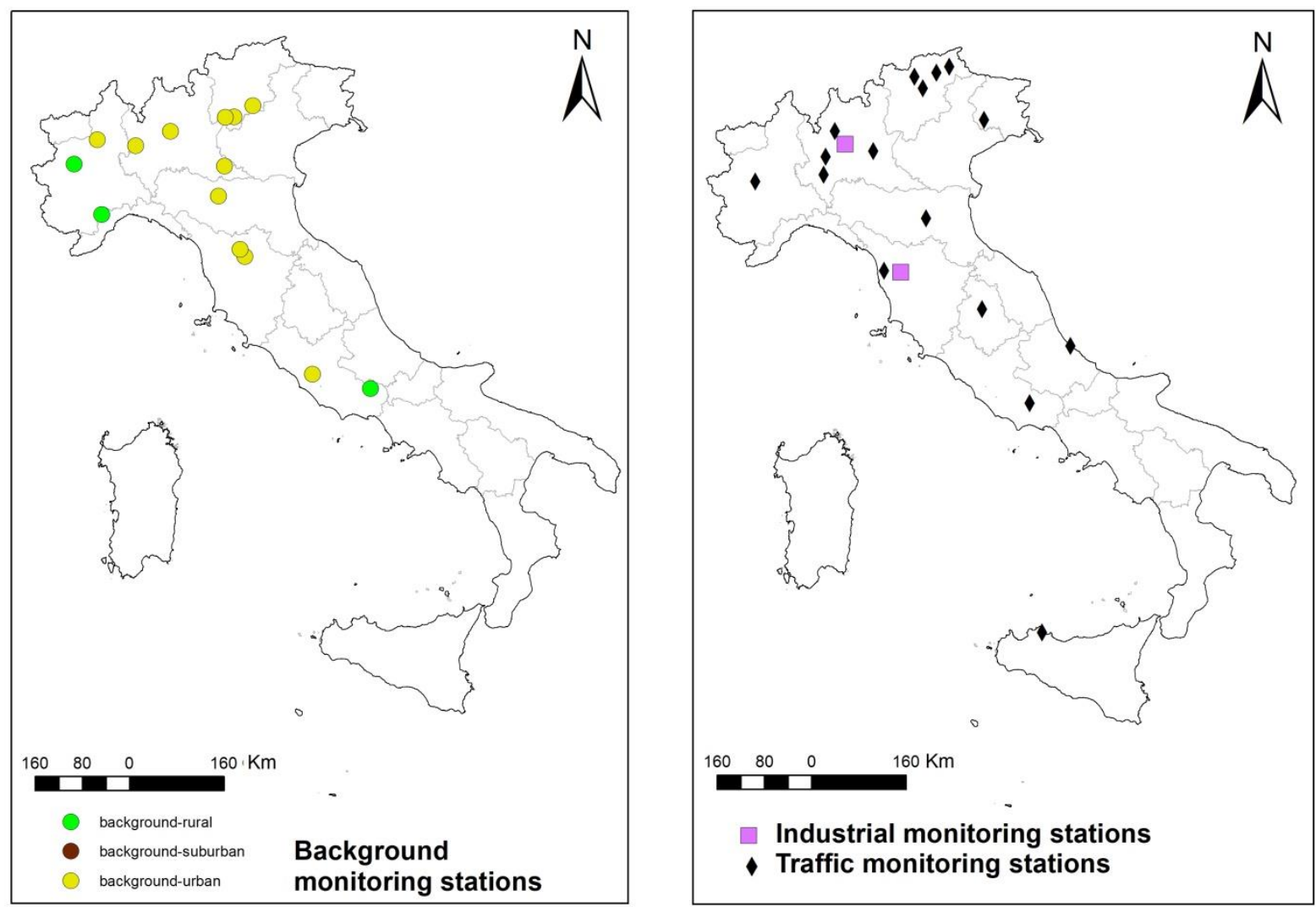

Figure S3 - Map of the Italian sites monitoring PM10 concentrations considered for the study. 

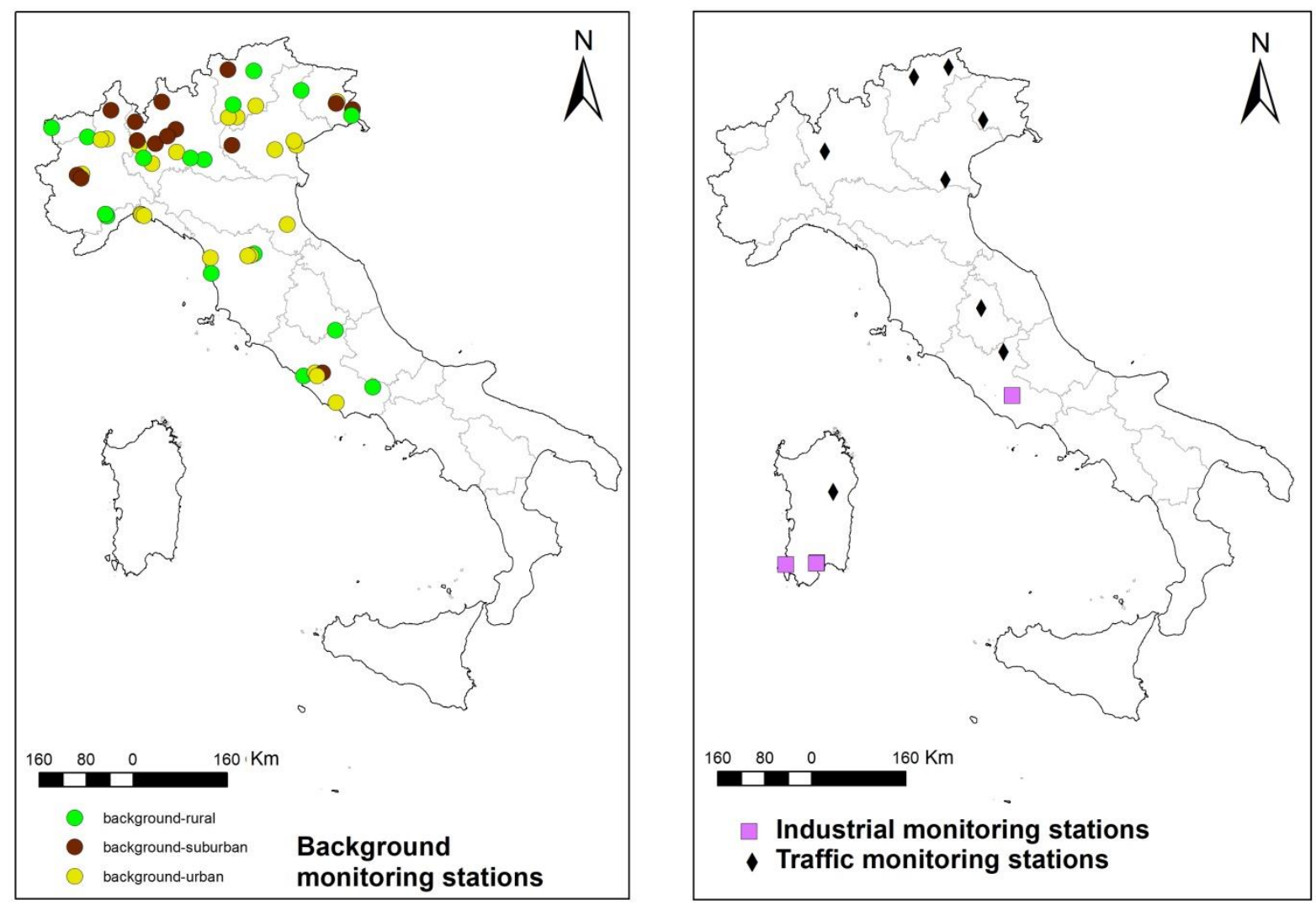

Figure S4 - Map of the Italian sites monitoring $\mathrm{O}_{3}$ concentrations considered for the study. 
Supplementary S2 - Emissions Time Series

Table S1 - SNAP Classification

\begin{tabular}{|l|l|}
\hline SNAP - macrosector \\
\hline SNAP code & Description \\
\hline 01 & Combustion in energy and transformation industries \\
\hline 02 & Non-industrial combustion plants \\
\hline 03 & Combustion in manufacturing industry \\
\hline 04 & Production processes \\
\hline 05 & Extraction and distribution of fossil fuels and geothermal energy \\
\hline 06 & Solvent use and other product use \\
\hline 07 & Road transport \\
\hline 08 & Other mobile sources and machinery \\
\hline 09 & Waste treatment and disposal \\
\hline 10 & Agriculture \\
\hline
\end{tabular}



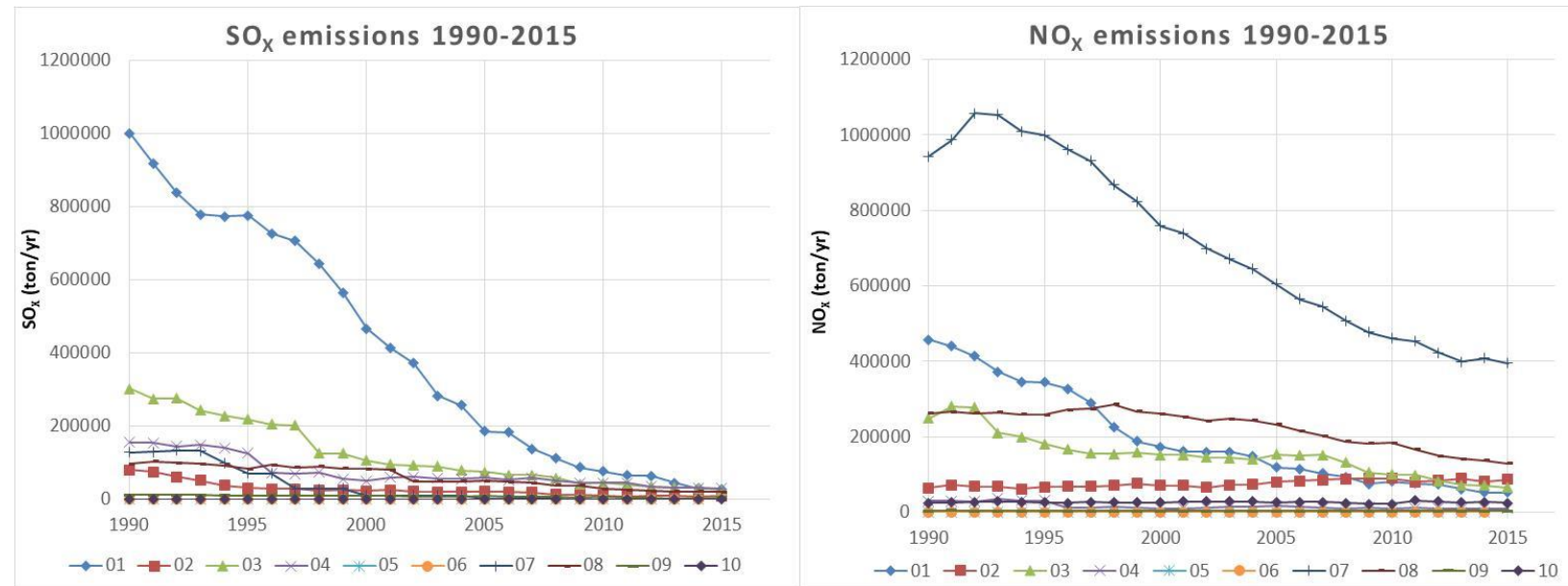

Figure S5 - Emission time series for $\mathrm{SO}_{\mathrm{X}}$ (on the left) and $\mathrm{NO}_{\mathrm{X}}$ (on the right) by SNAP sector for the period 1990-2015 elaborated for the national official submission 2017 used for the simulations of the MINNI model.
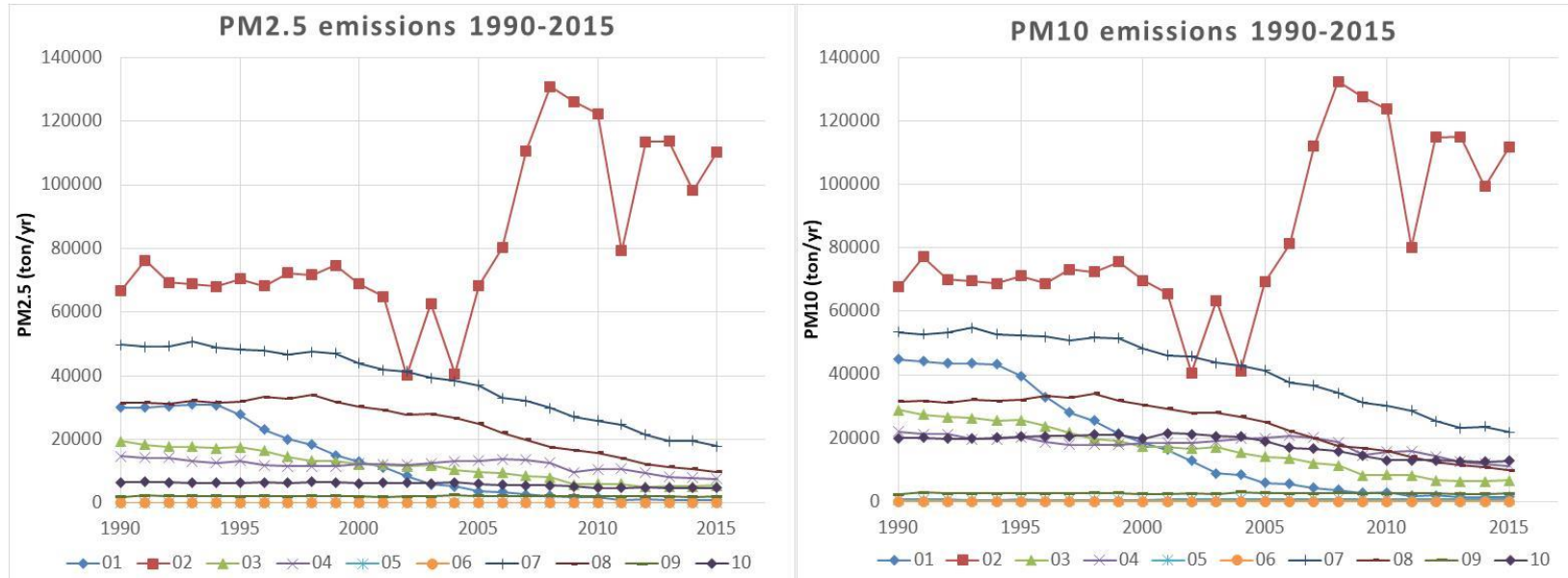

Figure S6 - Emission time series for PM2.5 (on the left) and PM10 (on the right) by SNAP sector for the period 1990-2015 elaborated for the national official submission 2017 used for the simulations of the MINNI model.
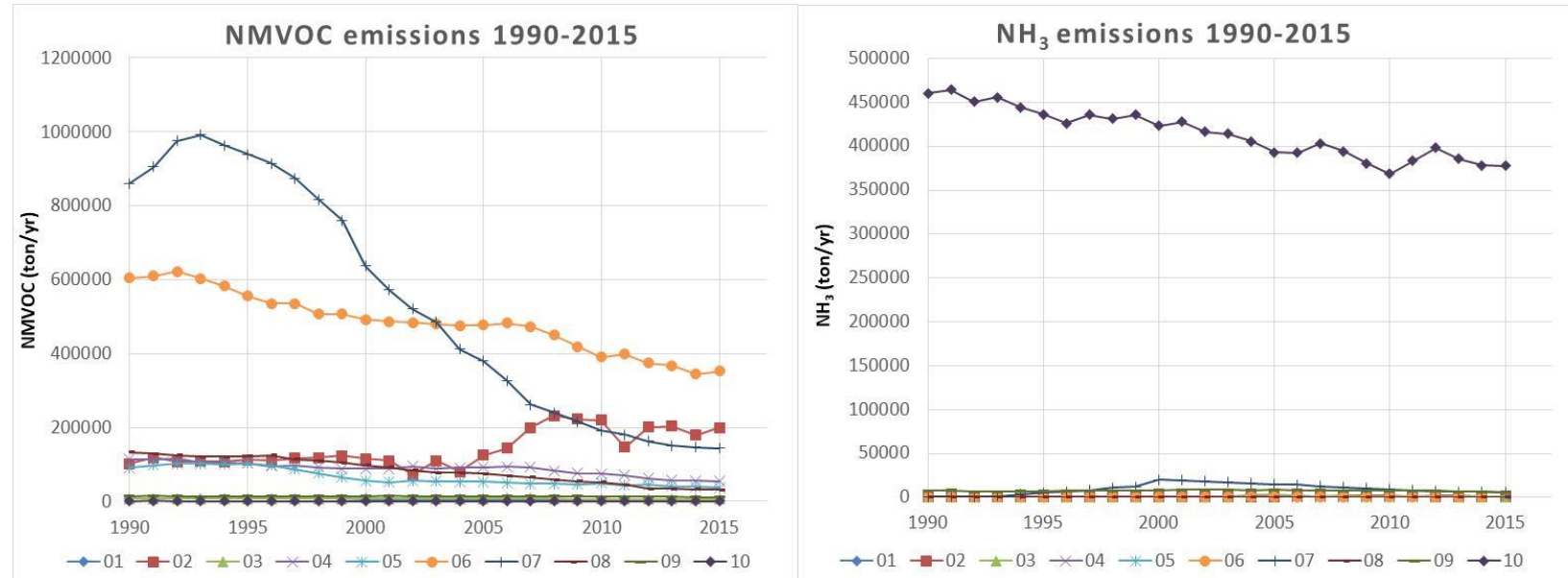

Figure S7 - Emission time series for NMVOC (on the left) and $\mathrm{NH}_{3}$ (on the right) by SNAP sector for the period 1990-2015 elaborated for the national official submission 2017 used for the simulations of the MINNI model. 


\section{S3.1 Temperature}
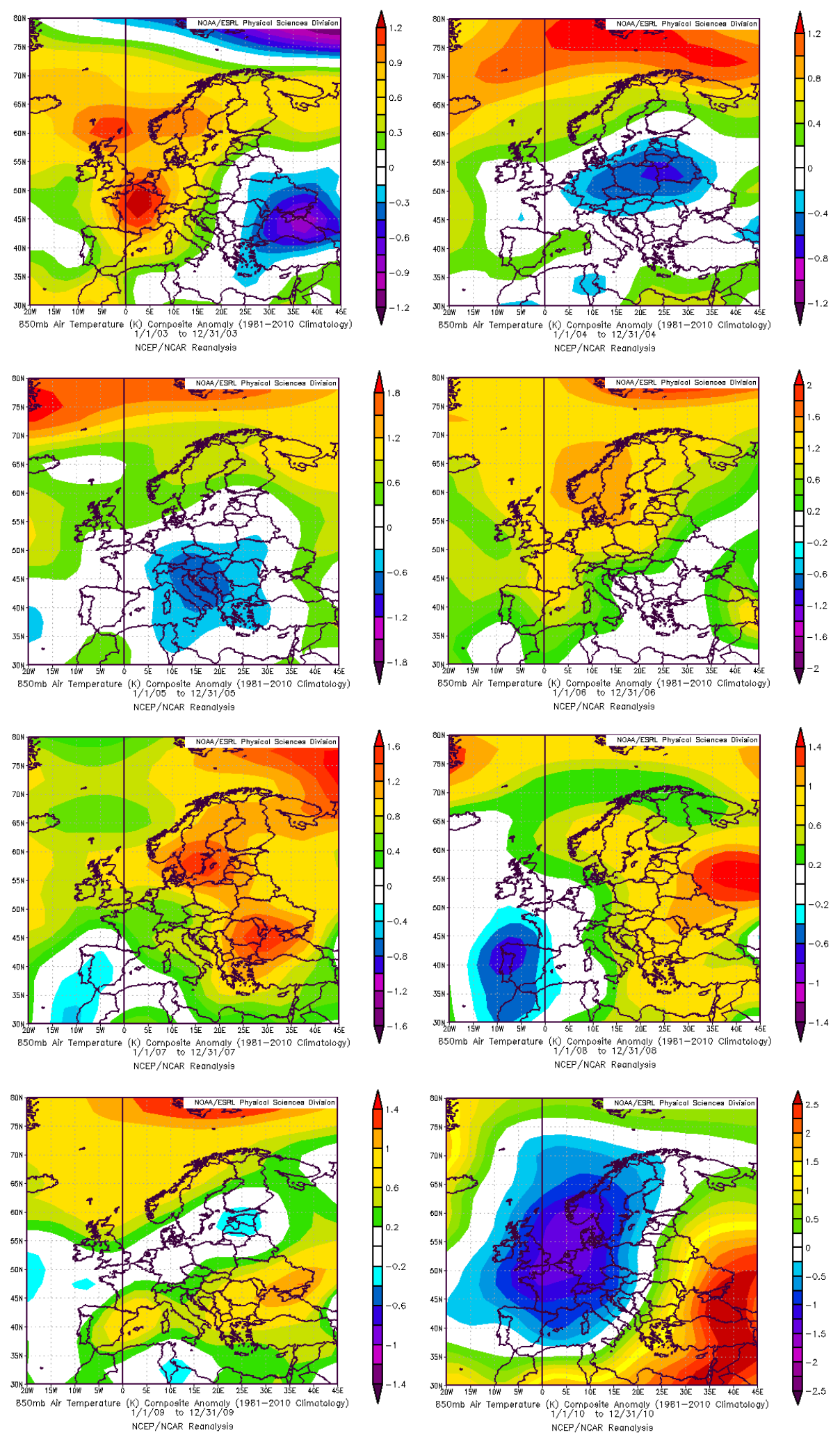

Figure S8 - Annual Temperature (850-hPa) anomalies elaborated from the NCEP/NCAR Reanalysis. Image provided by the NOAA/ESRL Physical Sciences Laboratory, Boulder Colorado from their Web site at http://psl.noaa.gov/. 
S3.2 500-hPa geopotential height
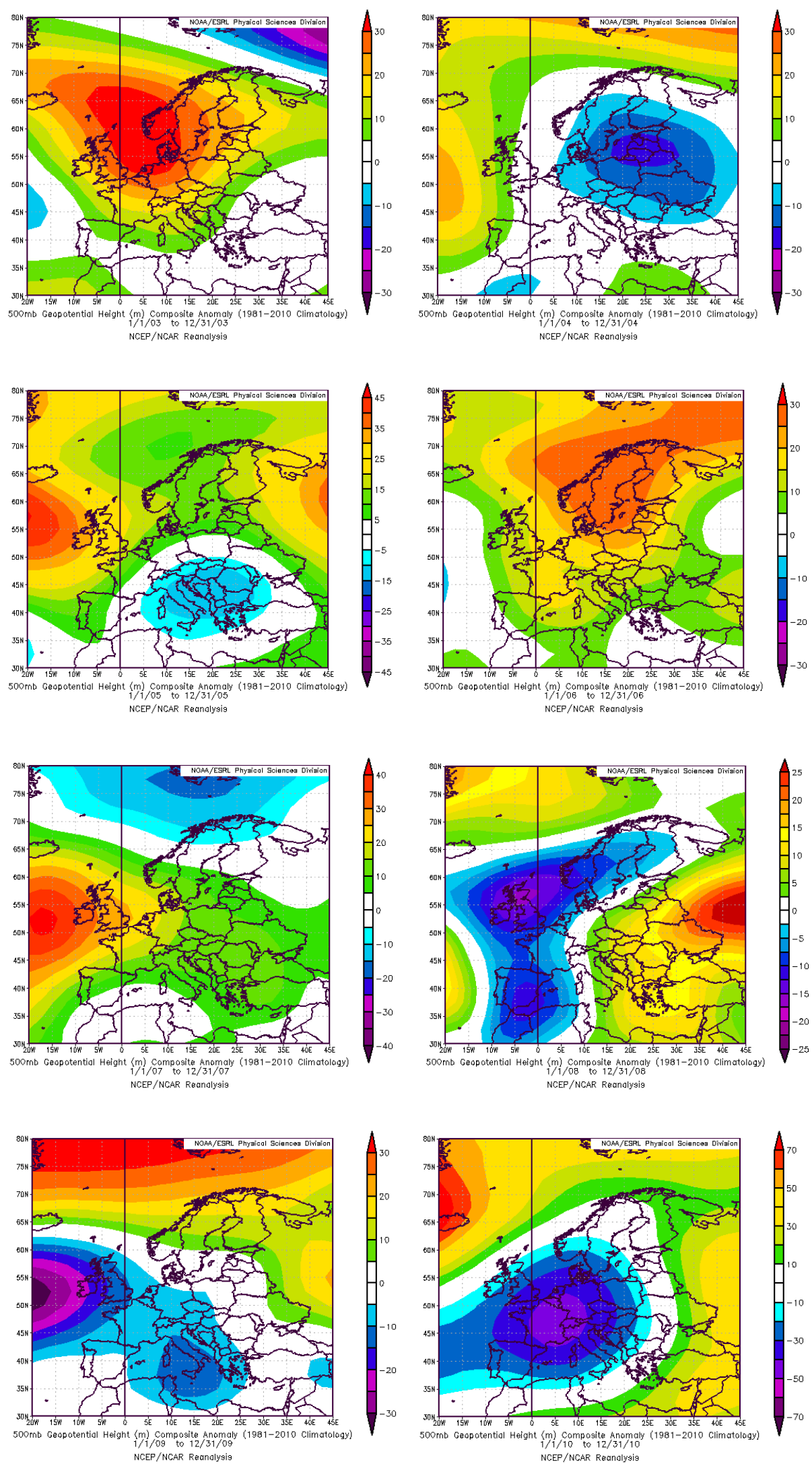

Figure S9 - Annual 500-hPa geopotential height anomalies elaborated from the NCEP/NCAR Reanalysis. Image provided by the NOAA/ESRL Physical Sciences Laboratory, Boulder Colorado from their Web site at http://psl.noaa.gov/. 
S3.3 Precipitation rate
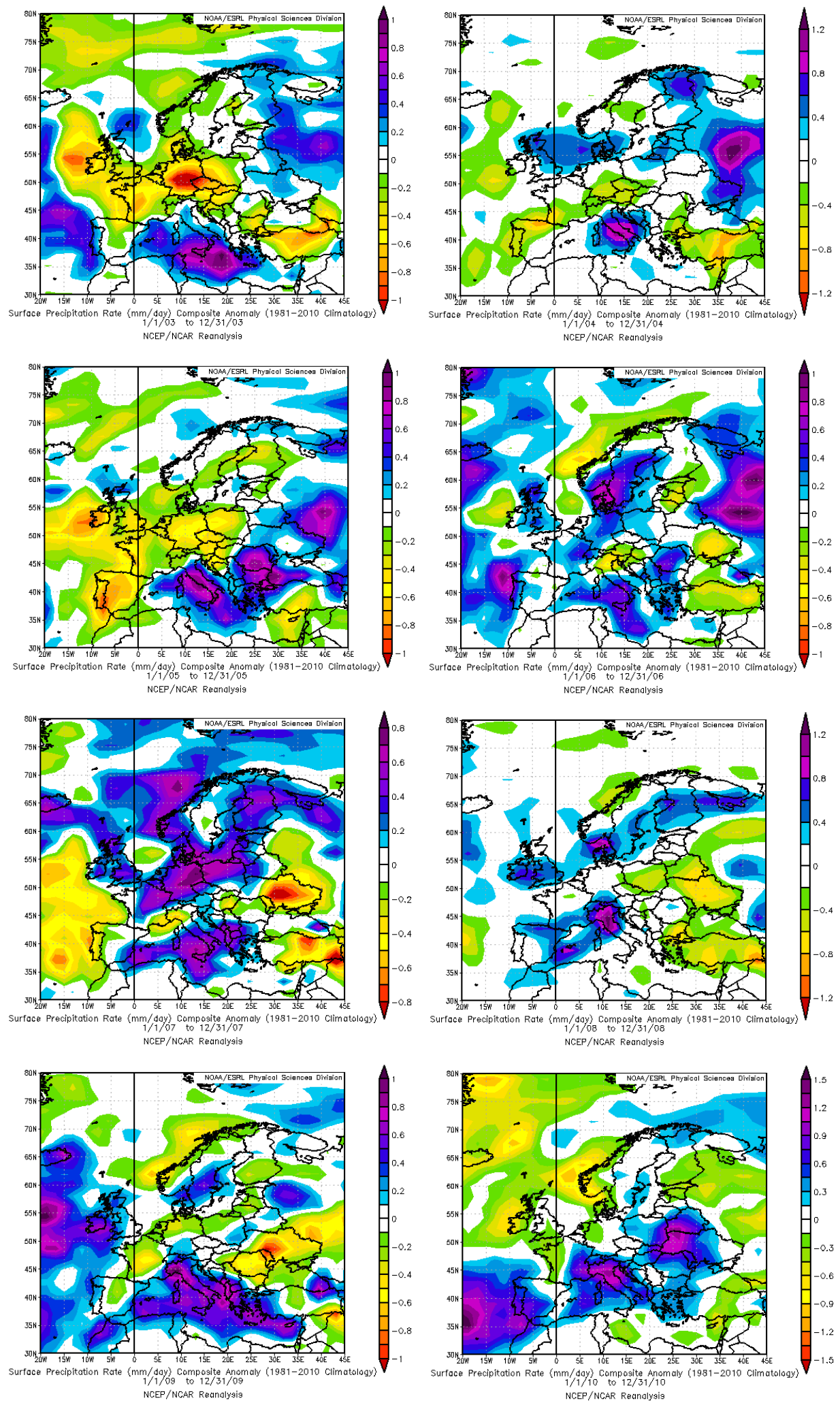

Figure S10 - Annual Precipitation rate anomalies elaborated from the NCEP/NCAR Reanalysis. Image provided by the NOAA/ESRL Physical Sciences Laboratory, Boulder Colorado from their Web site at http://psl.noaa.gov/. 

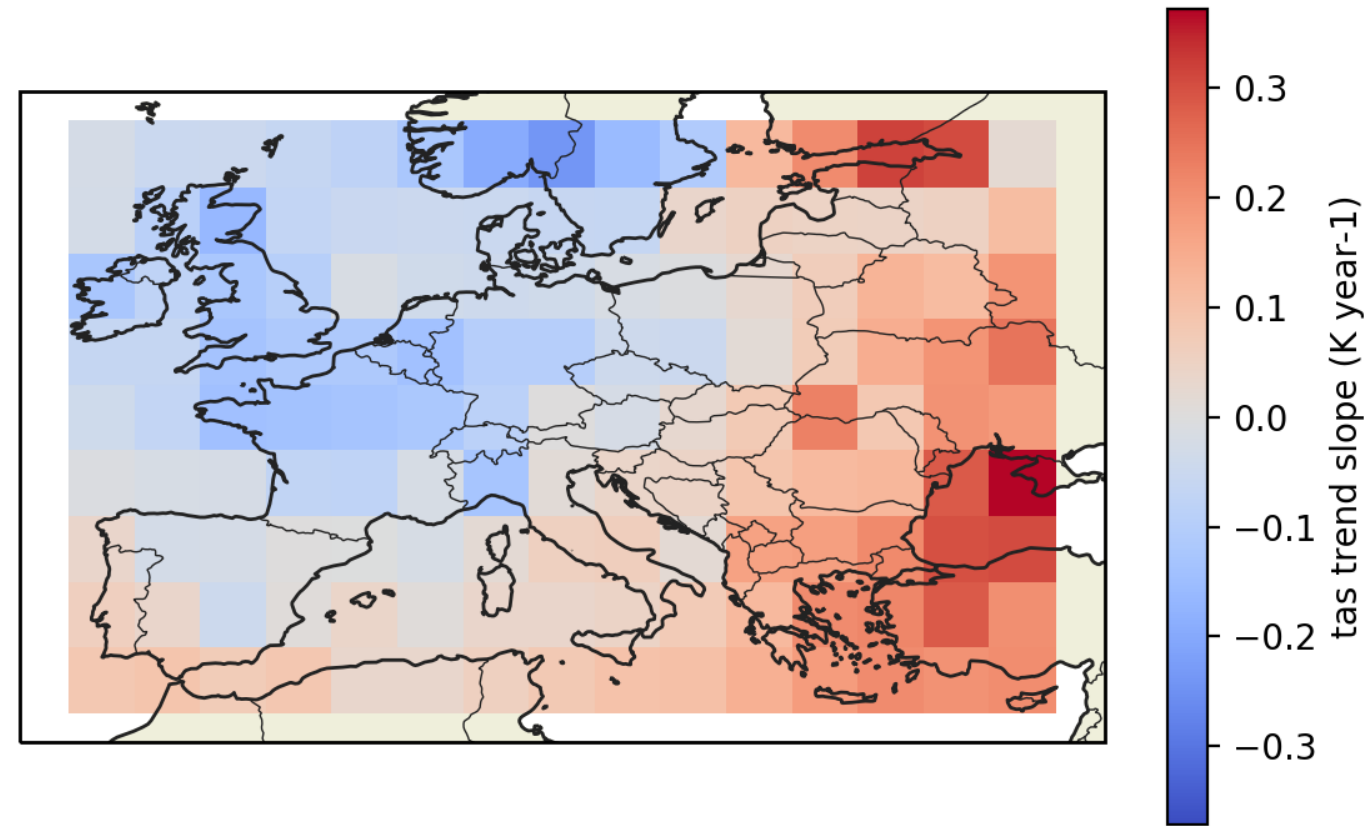

Figure S11 - Near surface temperature trend $\left(\mathrm{K} \mathrm{yr}^{-1}\right)$ for the period 2003-2010 computed from Copernicus Climate Data Service. 


\section{Supplementary S4 - Model simulation validation}

\section{S4.1 Definition of model evaluation statistics}

The performance statistical indexes used to compare modelled and observed values are calculated according to the following definitions

$\boldsymbol{M B}$ (Mean bias)

$$
\mathrm{MB}=\frac{1}{n} \sum_{i=1}^{n} M_{i}-O_{i}
$$

$\boldsymbol{R M S E}$ (Root mean squared error)

$$
R M S E=\left(\frac{\sum_{i=1}^{n}\left(M_{i}-O_{i}\right)^{2}}{n}\right)^{1 / 2}
$$

$\operatorname{corr}$ (correlation coefficient)

$$
\operatorname{corr}=\frac{1}{(n-1)} \sum_{i=1}^{n}\left(\frac{M_{i}-\bar{M}}{\sigma_{M}}\right)\left(\frac{O_{i}-\bar{O}}{\sigma_{O}}\right)
$$

where the letters $O$ and $M$ stand for observations and model results, respectively, the subscript $i$ indicates the time step, the overbars indicate the time average over $n$ time intervals, while the symbol $\sigma$ indicates the standard deviation.

\section{S4.2 Comparison of model performances with Colette et al. (2011)}

Table S2 - Model performances at all valid Italian monitoring stations computed over 8 years (2003-2010), as discussed in this

\begin{tabular}{|c|c|c|c|c|c|c|c|c|c|c|c|c|c|}
\hline & \multicolumn{3}{|c|}{ Daily $\mathrm{NO}_{2}$} & \multicolumn{3}{|c|}{ Daily PM10 } & \multicolumn{3}{|c|}{ Daily $\mathrm{O}_{3}$} & \multicolumn{3}{|c|}{$\mathrm{MDA8} \mathrm{O}_{3}$} \\
\hline & & $R M S E$ & $M B$ & corr & $R M S E$ & $M B$ & corr & $R M S E$ & $M B$ & corr & $R M S E$ & $M B$ & corr \\
\hline \multirow{5}{*}{ 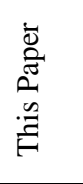 } & BKG, rural & 10.8 & -4.8 & 0.578 & 21.1 & -12.8 & 0.453 & 23.2 & 2.2 & 0.757 & 24.4 & -1.9 & 0.817 \\
\hline & BKG. suburban & 15.8 & -4.2 & 0.689 & & & & 21.7 & 9.3 & 0.814 & 25.0 & 5.1 & 0.847 \\
\hline & BKG. urban & 17.3 & -9.1 & 0.662 & 21.6 & -8.8 & 0.567 & 23.4 & 12.3 & 0.822 & 25.1 & 9.7 & 0.853 \\
\hline & IND & 18.7 & -7.9 & 0.606 & 17.3 & -3.9 & 0.630 & 25.7 & 18.6 & 0.683 & 25.0 & 14.8 & 0.712 \\
\hline & TRA & 28.6 & -22.4 & 0.591 & 22.5 & -12.2 & 0.585 & 25.6 & 17.8 & 0.761 & 25.1 & 13.4 & 0.801 \\
\hline \multirow{6}{*}{$\begin{array}{l}\bar{\pi} \\
\frac{\pi}{0} \\
\frac{0}{0} \\
\frac{0}{0} \\
0\end{array}$} & BOLCHEM & 12.6 & -1.46 & 0.658 & 13.9 & -4.48 & 0.370 & 17.6 & -4.31 & 0.782 & & & \\
\hline & CHIMERE & 16.6 & -13.4 & 0.633 & 15.5 & -11.3 & 0.576 & 23.5 & 18.5 & 0.797 & & & \\
\hline & EMEP & 17.4 & -14.1 & 0.574 & 17.9 & -14.4 & 0.542 & 21.0 & 13.3 & 0.740 & & & \\
\hline & EURAD & 16.0 & -6.46 & 0.644 & 15.1 & -3.75 & 0.524 & 25.2 & 15.0 & 0.659 & & & \\
\hline & OSLOCTM2 & 18.0 & -14.7 & 0.544 & & & & 23.5 & 17.1 & 0.750 & & & \\
\hline & MOZART & 21.3 & -18.1 & 0.350 & & & & 22.0 & 4.76 & 0.627 & & & \\
\hline
\end{tabular}
paper. Model performances at European suburban stations computed over 10 years (1998-2007) for the six chemistry-transport models, involved in Colette et al. (2011). 


\section{Supplementary S5 - Trend results}

\section{S5.1 $\mathrm{NO}_{2}$}

The following table shows the results for $\mathrm{NO}_{2}$ slopes for all the stations with a significant trend $(\mathrm{p}<0.05)$ per $\mathrm{Region}$, type and zone for the entire Italian territory. The rows in red show different behaviour between observed and modelled data (increasing trend in observations and decreasing one in simulations).

Table S3 - Results of observed and modelled trends for $\mathrm{NO}_{2}$ concentrations per stations with $\mathrm{p}<0.05$.

\begin{tabular}{|c|c|c|c|c|c|c|c|}
\hline $\begin{array}{l}\text { airbase } \\
\text { code }\end{array}$ & Region & Type & Zone & $\begin{array}{l}\text { range_obs } \\
\left(\mu \mathrm{g} \mathrm{m} \mathbf{m}^{-} \mathrm{yr}^{-1}\right)\end{array}$ & $\begin{array}{l}\text { range_sim } \\
\left(\mu \mathrm{g} \mathrm{m} \mathbf{-}^{-3} \mathrm{yr}^{-1}\right)\end{array}$ & $\begin{array}{c}\text { range\%_obs } \\
\left.\qquad \% \mathrm{yr}^{-1}\right)\end{array}$ & $\begin{array}{c}\text { range\%_sim } \\
\left(\% \mathrm{yr}^{-1}\right)\end{array}$ \\
\hline $499 \mathrm{~A}$ & ENTINO & ckground & burban & $-0.72)$ & $.17(-0.24--0.10)$ & 5.52) & $5.29--2.64)$ \\
\hline IT0507A & RENTINO & traffic & urban & $-1.00(-1.30--0.73)$ & $-1.21(-1.42--1.01)$ & $-2.81(-3.56--2.15)$ & $-6.27(-7.05--5.57)$ \\
\hline IT0508A & TRENTINO & traffic & urban & $-1.05(-1.45--0.70)$ & $-0.44(-0.52--0.38)$ & $-4.10(-5.39--2.89)$ & $-5.19(-5.86--4.64)$ \\
\hline IT0554A & PIEMONTE & background & urban & $-1.17(-1.89--0.44)$ & $-2.09(-2.63--1.64)$ & $-2.13(-3.25--0.82)$ & $-3.98(-4.79--3.20)$ \\
\hline IT0591A & TRENTINO & background & urban & $-0.84(-1.16--0.53)$ & $-0.75(-0.95--0.60)$ & $-2.40(-3.12--1.59)$ & $-3.43(-4.16--2.84)$ \\
\hline IT0702A & $\begin{array}{l}\text { EMILIA } \\
\text { ROMAGNA }\end{array}$ & traffic & urban & $-2.71(-3.20--2.23)$ & $-0.40(-0.64--0.20)$ & $-4.64(-5.20--3.93)$ & $-1.29(-2.03--0.67)$ \\
\hline IT0705A & LOMBARDIA & traffic & urban & $-0.92(-1.59--0.47)$ & $-1.14(-1.66--0.69)$ & $-1.55(-2.56--0.81)$ & $-1.79(-2.52--1.12)$ \\
\hline IT0770A & LOMBARDIA & backgrou & urban & $-1.22(-1.99--0.40)$ & $-0.91(-1.29--0.56)$ & $-2.04(-3.08--0.71)$ & $-1.68(-2.35--1.07)$ \\
\hline IT0771A & LOMBARDIA & traffic & urban & $-1.86(-2.51--1.09)$ & $-0.91(-1.18--0.69)$ & $-2.51(-3.30--1.52)$ & $-2.74(-3.42--2.16)$ \\
\hline IT0775A & LOMBARDIA & background & suburban & $-0.85(-1.32--0.39)$ & $-0.11(-0.22--0.03)$ & $-2.80(-4.12--1.37)$ & $-1.32(-2.44--0.38)$ \\
\hline IT0777A & LOMBARDIA & traffic & urban & $-1.61(-2.30--0.79)$ & $-0.77(-1.08--0.53)$ & $-2.61(-3.47--1.35)$ & $-2.31(-3.12--1.64)$ \\
\hline IT0839A & LOMBARDIA & background & urban & $-0.54(-0.90--0.17)$ & $-0.38(-0.62--0.22)$ & $-1.50(-2.37--0.48)$ & $-1.27(-2.00--0.74)$ \\
\hline IT0854A & LIGURIA & background & urban & $-1.37(-2.17--0.52)$ & $-1.64(-1.87--1.40)$ & $-2.83(-4.11--1.17)$ & $-4.31(-4.79--3.78)$ \\
\hline IT0861A & TOSCANA & traffic & urban & $4.26(3.29-5.22)$ & $-0.61(-0.97--0.32)$ & $6.57(4.82-8.82)$ & $-2.23(-3.32--1.22)$ \\
\hline IT0912A & LOMBARDIA & background & urban & $1.19(0.67-1.75)$ & $-0.88(-1.09--0.68)$ & $3.27(1.77-5.09)$ & $-2.38(-2.88--1.90)$ \\
\hline IT0963A & VENETO & background & ban & $1.17(-1.64--0.61)$ & $-1.24(-1.46--0.99)$ & $-2.99(-4.11--1.67)$ & $-2.98(-3.38--2.43)$ \\
\hline IT0966A & $\begin{array}{l}\text { FRIULI } \\
\text { VENEZIA } \\
\text { GIULIA }\end{array}$ & ound & ban & -0.54 & 8) & 2) & -4.7 \\
\hline ITOS & $\begin{array}{l}\text { VALLE } \\
\text { D'AOSTA }\end{array}$ & $\mathrm{d}$ & ral & -0 & 4) & $\begin{array}{c}-10.04(-12.29-- \\
6.68) \\
\end{array}$ & 18) \\
\hline IT1005A & LIGURIA & traffic & urban & $-1.01(-1.84--0.11)$ & $-0.97(-1.11--0.84)$ & $-2.07(-3.57--0.23)$ & $-4.96(-5.42--4.39)$ \\
\hline IT1010A & LOMBARDIA & background & urban & $-1.65(-2.55--0.87)$ & $-0.75(-1.03--0.42)$ & $-3.26(-4.61--1.83)$ & $-1.83(-2.41--1.05)$ \\
\hline IT1098A & $\begin{array}{l}\text { FRIULI } \\
\text { VENEZIA } \\
\text { GIULIA }\end{array}$ & traffic & suburban & $-1.06(-1.83--0.20)$ & $-0.81(-0.96--0.68)$ & $-2.49(-3.86--0.49)$ & $-3.76(-4.30--3.27)$ \\
\hline IT1104A & LOMBARDIA & affic & urban & $.20--3.77)$ & $-1.00(-1.23--0.76)$ & $-6.95(-8.13--5.69)$ & $-2.64(-3.20--2.07)$ \\
\hline IT1120A & PIEMONTE & backgro & suburba & $-0.91(-1.41--0.51)$ & $-1.98(-2.31--1.63)$ & $-2.07(-3.02--1.23)$ & $-4.73(-5.26--4.02)$ \\
\hline IT1125A & PIEMONTE & traffic & urban & $-1.06(-1.54--0.48)$ & $-1.57(-1.93--1.22)$ & $-2.50(-3.46--1.20)$ & $-4.36(-5.16--3.51)$ \\
\hline IT1143A & LIGURIA & traffic & urban & $-0.76(-1.13--0.34)$ & $-1.48(-1.67--1.32)$ & $-2.14(-3.09--1.01)$ & $-5.35(-5.76--4.88)$ \\
\hline IT1153A & LOMBARDIA & traffic & urban & $-0.99(-1.35--0.68)$ & $-0.54(-0.72--0.44)$ & $-2.48(-3.27--1.75)$ & $-1.99(-2.55--1.65)$ \\
\hline IT1174A & LOMBARDIA & background & rural & $-1.01(-1.34--0.68)$ & $-0.50(-0.73--0.30)$ & $-2.91(-3.74--2.04)$ & $-1.70(-2.41--1.04)$ \\
\hline IT1176A & LAZIO & background & urban & $-0.93(-1.47--0.40)$ & $-1.55(-1.90--1.20)$ & $-1.71(-2.63--0.76)$ & $-2.95(-3.47--2.38)$ \\
\hline IT1177A & VENETO & background & urban & $-1.37(-1.66--1.06)$ & $-0.67(-0.96--0.39)$ & $-3.13(-3.69--2.52)$ & $-2.11(-2.92--1.28)$ \\
\hline IT1203A & LOMBARDIA & background & suburban & $-0.95(-1.32--0.59)$ & $-1.02(-1.39--0.70)$ & $-2.66(-3.56--1.72)$ & $-2.51(-3.29--1.77)$ \\
\hline IT1207A & SICILIA & traffic & urban & $-2.85(-3.60--1.73)$ & $-1.48(-1.75--1.22)$ & $-4.66(-5.60--3.08)$ & $-6.57(-7.47--5.65)$ \\
\hline IT1246A & PIEMONTE & background & urban & $-0.83(-1.18--0.50)$ & $-0.59(-0.78--0.46)$ & $-2.66(-3.57--1.68)$ & $-4.15(-5.21--3.39)$ \\
\hline IT1247A & PIEMONTE & background & urban & $-0.55(-1.03--0.12)$ & $-0.52(-0.68--0.37)$ & $-1.61(-2.79--0.37)$ & $-3.63(-4.50--2.65)$ \\
\hline IT1286A & LOMBARDIA & traffic & urban & $-1.44(-2.25--0.71)$ & $-0.48(-0.79--0.26)$ & $-2.88(-4.14--1.52)$ & $-1.49(-2.34--0.83)$ \\
\hline IT1335A & TRENTINO & traffic & urban & $-0.52(-0.88--0.20)$ & $-0.78(-0.95--0.60)$ & $-1.41(-2.27--0.56)$ & $-5.38(-6.25--4.41)$ \\
\hline IT1336A & VENETO & traffic & urban & $-3.27(-3.87--2.74)$ & $-0.59(-0.81--0.37)$ & $-5.28(-5.95--4.68)$ & $-1.91(-2.54--1.25)$ \\
\hline IT1409A & TOSCANA & traffic & urban & $-0.81(-1.15--0.47)$ & $-0.57(-0.68--0.45)$ & $-1.82(-2.49--1.10)$ & $-2.59(-3.04--2.13)$ \\
\hline IT1422A & ABRUZZO & traffic & urban & $-2.01(-3.24--0.43)$ & $-1.26(-1.43--1.10)$ & $-2.51(-3.72--0.58)$ & $-6.08(-6.69--5.57)$ \\
\hline IT1453A & VENETO & background & urban & $-1.36(-1.81--0.84)$ & $-0.89(-1.11--0.71)$ & $-2.78(-3.45--1.82)$ & $-2.45(-2.99--2.01)$ \\
\hline IT1459A & LOMBARDIA & background & suburban & $-0.95(-1.46--0.37)$ & $-1.32(-1.70--0.97)$ & $-2.31(-3.35--0.96)$ & $-3.16(-3.92--2.43)$ \\
\hline IT1463A & LOMBARDIA & background & suburban & $0.57(0.10-1.16)$ & $-0.33(-0.72--0.01)$ & $2.01(0.34-4.42)$ & $-0.79(-1.69--0.02)$ \\
\hline
\end{tabular}




\section{S5.2 PM10}

The following table shows the results for PM10 slopes for all the stations with a significant trend $(\mathrm{p}<0.05)$ per Region, type and zone for the entire Italian territory.

Table S4 - Results of observed and modelled trends for PM10 concentrations per stations with $\mathrm{p}<0.05$.

\begin{tabular}{|c|c|c|c|c|c|c|c|}
\hline $\begin{array}{l}\text { airbase_ } \\
\text { code }\end{array}$ & Region & Type & Zone & $\begin{array}{c}\text { range_obs } \\
\left(\mu \mathrm{g} \mathrm{m}^{-3} \mathrm{yr}^{-1}\right)\end{array}$ & $\begin{array}{c}\text { range_sim } \\
\left(\mu \mathrm{g} \mathrm{m}-{ }^{3} \mathrm{yr}^{-1}\right)\end{array}$ & $\begin{array}{c}\text { range\%_obs } \\
\left(\% \mathbf{y r}^{-1}\right)\end{array}$ & $\begin{array}{l}\text { range\%_sim } \\
\left(\% \mathrm{yr}^{-1}\right)\end{array}$ \\
\hline IT0559A & LOMBARDIA & industrial & suburban & $-2.59(-3.14--2.17)$ & $-0.81(-1.37--0.20)$ & $-5.08(-5.88--4.43)$ & $-2.11(-3.38--0.55)$ \\
\hline IT0705A & LOMBARDIA & traffic & urban & $-1.85(-2.30--1.44)$ & $-1.26(-2.04--0.48)$ & $-3.42(-4.16--2.71)$ & $-2.36(-3.58--0.94)$ \\
\hline IT0707A & LOMBARDIA & background & urban & $-2.25(-2.80--1.68)$ & $-0.98(-1.53--0.42)$ & $-4.53(-5.38--3.53)$ & $-2.61(-3.92--1.19)$ \\
\hline IT0709A & LOMBARDIA & background & urban & $-1.99(-2.83--1.09)$ & $-0.94(-1.41--0.37)$ & $-3.69(-4.97--2.18)$ & $-2.75(-3.87--1.16)$ \\
\hline IT0776A & LOMBARDIA & traffic & urban & $-1.86(-2.23--1.44)$ & $-0.79(-1.09--0.47)$ & $-4.56(-5.23--3.71)$ & $-3.26(-4.28--2.08)$ \\
\hline IT0940A & EMILIA ROMAGNA & background & urban & $-0.72(-1.21--0.05)$ & $-1.19(-1.64--0.67)$ & $-1.99(-3.24--0.14)$ & $-3.36(-4.45--2.05)$ \\
\hline IT0953A & LAZIO & background & urban & $-0.60(-0.98--0.11)$ & $-0.55(-0.86--0.09)$ & $-1.98(-3.04--0.37)$ & $-1.90(-2.85--0.31)$ \\
\hline IT1079A & SICILIA & traffic & urban & $-0.88(-1.26--0.45)$ & $-0.93(-1.17--0.66)$ & $-2.22(-3.06--1.17)$ & $-3.91(-4.68--2.96)$ \\
\hline IT1104A & LOMBARDIA & traffic & urban & $-1.56(-2.11--1.00)$ & $-1.31(-1.78--0.84)$ & $-3.24(-4.22--2.14)$ & $-3.55(-4.59--2.41)$ \\
\hline IT1159A & EMILIA ROMAGNA & traffic & urban & $-1.21(-1.70--0.82)$ & $-0.65(-1.18--0.16)$ & $-2.74(-3.63--1.94)$ & $-2.08(-3.48--0.55)$ \\
\hline IT1247A & PIEMONTE & background & urban & $-1.51(-1.98--1.02)$ & $-0.68(-1.09--0.21)$ & $-4.15(-5.09--3.00)$ & $-3.48(-5.20--1.20)$ \\
\hline
\end{tabular}




\section{$\mathrm{S5.3} \mathrm{O}_{3}$}

The following table shows the results for $\mathrm{O}_{3}$-MDA8 (Apr/Sep) slopes for all the stations with a significant trend $(\mathrm{p}<0.05)$ per Region, type and zone for the entire Italian territory. The rows in red show different behaviour between observed and modelled data (increasing trend in observations and decreasing one in simulations).

Table S5 - Results of observed and modelled trends for $\mathrm{O}_{3}$-MDA8 (Apr/Sep) concentrations per stations with $\mathrm{p}<0.05$.

\begin{tabular}{|c|c|c|c|c|c|c|c|}
\hline $\begin{array}{l}\text { airbase } \\
\text { code }\end{array}$ & Region & Type & Zone & $\begin{array}{l}\text { range_obs } \\
\left(\mu \mathrm{g} \mathrm{m}^{-}{ }^{-} \mathrm{yr}^{-1}\right)\end{array}$ & $\underset{\left(\mu \mathrm{g} \mathrm{m}^{-}{ }^{-} \mathrm{yr}^{-1}\right)}{\text { rangesim }}$ & $\begin{array}{c}\text { range\%_obs } \\
\left(\% \mathrm{yr}^{-1}\right)\end{array}$ & $\begin{array}{c}\text { range\%_sim } \\
\left(\% \mathrm{yr}^{-1}\right)\end{array}$ \\
\hline IT0499A & TRENTINO & ackground & suburban & $1.10(0.21-2.10)$ & $-0.67(-1.01--0.56)$ & $1.33(0.24-2.68)$ & $-0.79(-1.18--0.66)$ \\
\hline IT0505A & TRENTINO & background & rural & $-1.45(-1.91--0.98)$ & $-0.30(-0.71--0.17)$ & $-1.13(-1.47--0.78)$ & $-0.33(-0.77--0.19)$ \\
\hline IT0508A & TRENTINO & traffic & urban & $1.54(1.06-2.01)$ & $-0.56(-0.84--0.38)$ & $1.98(1.34-2.63)$ & $-0.63(-0.94--0.43)$ \\
\hline IT0858A & LIGURIA & background & urban & $2.07(1.05-3.59)$ & $-0.49(-0.97--0.03)$ & $2.12(1.03-3.92)$ & $-0.40(-0.78--0.02)$ \\
\hline IT0867A & LAZIO & traffic & urban & $3.67(2.20-5.88)$ & $-0.79(-1.23--0.50)$ & $4.82(2.58-9.30)$ & $-0.75(-1.14--0.48)$ \\
\hline IT0883A & TOSCANA & background & rural & $-1.08(-1.70--0.46)$ & $-0.79(-1.22--0.40)$ & $-0.95(-1.46--0.42)$ & $-0.69(-1.04--0.35)$ \\
\hline IT0888A & LAZIO & industrial & suburban & $-3.27(-3.98--2.36)$ & $-1.06(-1.70--0.52)$ & $-3.26(-3.87--2.41)$ & $-0.94(-1.48--0.47)$ \\
\hline IT0948A & TOSCANA & background & urban & $1.19(0.27-2.05)$ & $-0.70(-1.22--0.24)$ & $1.18(0.26-2.14)$ & $-0.60(-1.02--0.21)$ \\
\hline IT0953A & LAZIO & background & urban & $-3.39(-4.54--2.44)$ & $-1.40(-2.34--0.73)$ & $-3.00(-3.87--2.24)$ & $-1.23(-1.99--0.66)$ \\
\hline IT0988A & $\begin{array}{l}\text { VALLE } \\
\text { D'AOSTA }\end{array}$ & background & rural & $-4.64(-6.23--2.40)$ & $-0.62(-1.17--0.19)$ & $-3.80(-4.82--2.17)$ & $-0.65(-1.21--0.20)$ \\
\hline IT0989A & LAZIO & background & rural & $-6.92(-7.64--6.45)$ & $-0.58(-0.71--0.47)$ & $-4.31(-4.69--4.11)$ & $-0.60(-0.74--0.49)$ \\
\hline IT1017A & LOMBARDIA & background & suburban & $-2.51(-3.41--1.58)$ & $-1.74(-2.84--0.93)$ & $-2.05(-2.74--1.34)$ & $-1.42(-2.25--0.78)$ \\
\hline IT1048A & $\begin{array}{l}\text { EMILIA } \\
\text { ROMAGNA }\end{array}$ & background & urban & $-2.60(-3.57--1.55)$ & $-1.43(-2.10--0.85)$ & $-2.28(-3.05--1.40)$ & $-1.20(-1.72--0.73)$ \\
\hline IT1110A & TOSCANA & background & urban & $-0.86(-1.36--0.21)$ & $-1.15(-1.65--0.68)$ & $-0.86(-1.34--0.22)$ & $-0.94(-1.32--0.57)$ \\
\hline IT1174A & LOMBARDIA & background & rural & $-3.52(-5.04--2.32)$ & $-0.66(-1.63--0.09)$ & $-2.74(-3.74--1.87)$ & $-0.54(-1.31--0.08)$ \\
\hline IT1182A & UMBRIA & traffic & urban & $1.42(0.68-2.23)$ & $-0.79(-1.23--0.36)$ & $1.53(0.71-2.51)$ & $-0.73(-1.11--0.34)$ \\
\hline IT1191A & TRENTINO & background & rural & $-1.62(-2.89--0.20)$ & $-0.70(-1.18--0.30)$ & $-1.24(-2.17--0.16)$ & $-0.68(-1.12--0.30)$ \\
\hline IT1203A & LOMBARDIA & background & suburban & $-4.81(-5.80--4.14)$ & $-0.94(-1.90--0.16)$ & $-3.33(-3.90--2.91)$ & $-0.78(-1.52--0.14)$ \\
\hline IT1216A & $\begin{array}{l}\text { FRIULI } \\
\text { VENEZIA } \\
\text { GIULIA }\end{array}$ & background & suburban & $2.40(1.71-3.72)$ & $-0.89(-1.65--0.29)$ & $2.94(2.01-4.86)$ & $-0.76(-1.38--0.25)$ \\
\hline IT1236A & TOSCANA & background & rural & $-5.29(-5.98--4.32)$ & $-0.79(-1.41--0.24)$ & $-4.13(-4.62--3.50)$ & $-0.65(-1.14--0.20)$ \\
\hline IT1246A & PIEMONTE & background & urban & $-2.03(-3.16--0.89)$ & $-0.67(-1.27--0.21)$ & $-1.63(-2.46--0.74)$ & $-0.60(-1.13--0.19)$ \\
\hline IT1247A & PIEMONTE & background & urban & $-4.38(-5.38--3.40)$ & $-0.94(-1.50--0.50)$ & $-3.36(-3.95--2.68)$ & $-0.87(-1.36--0.47)$ \\
\hline IT1279A & SARDEGNA & industrial & suburban & $-2.45(-3.66--1.31)$ & $-0.84(-1.32--0.43)$ & $-2.32(-3.34--1.29)$ & $-0.73(-1.14--0.38)$ \\
\hline IT1397A & SARDEGNA & industrial & suburban & $-1.80(-2.99--0.47)$ & $-0.87(-1.31--0.52)$ & $-1.81(-2.90--0.49)$ & $-0.77(-1.13--0.46)$ \\
\hline IT1427A & SARDEGNA & traffic & urban & $-1.51(-2.72--0.66)$ & $-0.38(-0.50--0.35)$ & $-1.60(-2.76--0.74)$ & $-0.40(-0.51--0.36)$ \\
\hline IT1474A & $\begin{array}{l}\text { FRIULI } \\
\text { VENEZIA } \\
\text { GIULIA }\end{array}$ & background & rural & $2.61(0.78-4.60)$ & $-0.79(-1.49--0.19)$ & $2.55(0.71-4.93)$ & $-0.67(-1.24--0.17)$ \\
\hline IT1478A & $\begin{array}{l}\text { FRIULI } \\
\text { VENEZIA } \\
\text { GIULIA }\end{array}$ & background & rural & $-3.83(-5.57--2.17)$ & $-0.56(-1.21--0.13)$ & $-3.17(-4.39--1.93)$ & $-0.55(-1.16--0.13)$ \\
\hline IT1515A & PIEMONTE & background & suburban & $-3.23(-4.45--1.82)$ & $-0.66(-0.81--0.58)$ & $-2.61(-3.50--1.54)$ & $-0.68(-0.83--0.60)$ \\
\hline IT1519A & PIEMONTE & background & rural & $-2.84(-3.34--2.41)$ & $-1.14(-1.59--0.86)$ & $-2.17(-2.53--1.89)$ & $-1.00(-1.39--0.76)$ \\
\hline IT1576A & SARDEGNA & industrial & suburban & $2.29(0.89-3.37)$ & $-1.04(-1.46--0.57)$ & $2.96(1.08-4.80)$ & $-0.86(-1.19--0.48)$ \\
\hline
\end{tabular}

